\title{
A Dynamic Planning Method for Mobile Agent Implementation based on Service Recommendation
}

\author{
Xiaolin Wang* and Guangzhou Zeng \\ School of Computer Science and Technology, Shandong University, Jinan 250101, P. R. China
}

Received: 10 Jun. 2013, Revised: 15 Oct. 2013, Accepted: 16 Oct. 2013

Published online: 1 May. 2014

\begin{abstract}
Mobile agent is a task proxy of the social member that is able to migrate continuously among hosts and use the local services to complete tasks. The hosts, known as workplaces, can be regarded as service proxies of other social members. The sequence of workplaces on which mobile agent completes its tasks is called path. The main contribution of this paper is proposed a dynamic planning method for mobile agent path based on the variable decision space. The path planning model is described with MDP, and the variable decision space is generated through social acquaintance recommendation, assuming that every social member can provide workplace and service recommendation for mobile agent in cooperative work manner. The experimentation results show that by utilizing social acquaintance recommendation, mobile agent is able to dynamically construct its path based on variable recommended space.
\end{abstract}

Keywords: Mobile agent, dynamic path planning, markov decision process, social acquaintance recommendation, variable decision space.

\section{Introduction}

Mobile agent is a task proxy of the social member that is able to migrate continuously among hosts and use local service of hosts to perform tasks. The hosts, known as workplaces, can be regarded as service proxies of other social members. The sequence of workplaces on which mobile agent completes tasks is called path. Path planning has been one of the key issues in mobile agent application.

Mobile agent path planning methods can be divided into static planning and dynamic planning [1,2]. The former refers to approaches that the path has been created before mobile agent launching. The latter refers to approaches that mobile agent has no information about the path before launching, and the workplace must be dynamically decided at every moving stage based on task to be executed and knowledge of environment.

The most commonly used static planning method is itinerary design $[3,4,5]$. Since information of workplaces on the path is the prior knowledge about environment, it cannot adapt well to the environment changes, such as network disconnection, service change, host failure, etc.

Reference $[6,7,8,9,10]$ gave a kind of dynamic path planning methods based on service discovery, in which mobile agent detects environment at first, and then evaluates and selects workplace every time it migrates. As compared with itinerary designing, the service discovery mechanism has more flexibility to environment, while it requires mobile agent to carry sufficient knowledge and coded function to service discovery. Therefore it leads to a larger size of mobile agent itself. As well known, the larger the mobile agent is, the longer migrating time it takes, and the migration failure probability will increase.

Considering the lightweight of mobile agent, reference [11] proposed a navigation method for mobile agent path planning. The main idea of navigation mechanism is dividing the whole environment into several navigating domains, where a navigating station is located at each domain and all the navigating stations are organized as a navigating tree. By tree style navigating, mobile agent is able to dynamically plan its path. However, prior knowledge about the whole environment is necessary for building the navigating tree, therefore this approach cannot adapt well to open environment.

Continuing the previous work [12], this paper gives a dynamic planning method of mobile agent path based on MDP (Markov Decision Process) and social acquaintance recommendation, assuming that every social member can provide workplace and acquaintance recommendation for

\footnotetext{
*Corresponding author e-mail: xlwang@sdu.edu.cn
} 
mobile agent in accordance with their cooperative contracts. Acquaintance recommendation mechanism is similar to the concept of navigation, but it is more suitable for mobile agent working in an open environment, since it does not rely on the prior knowledge about the whole environment.

The structure of the following parts of this paper is: Section 2 gives the definition of mobile agent path; in Section 3 the MDP model of path planning is built; Section 4 discusses the social acquaintance recommendation mechanism; Section 5 describes the path planning algorithms; Section 6 illustrates the experiment results; Section 7 is about discussion on related work; a summary and a prospect about future work is shown in the final section.

\section{Definition of mobile agent path}

To simplify the discussion, this paper prescribes: (1) Tspec $=\left(\text { task }_{i}\right)_{i=1}^{N}$ is a sequence of tasks ${ }^{1}$; (2) $\forall t_{\text {task }}$, task $_{i+1} \in T$ spec, task $_{i}$ and task $_{i+1}$ are executed at two adjacent workplaces $w p_{i}$ and $w p_{i+1}$ respectively ${ }^{2}$; (3) $w p_{0}$ is a special place where mobile agent was launched.

Definition 2.1 Mobile agent is defined as $M A=($ aid,$T$ spec, aLog, aEng $)$, where

- aid is the mobile agent identifier;

- Tspec is a sequence of tasks and $\forall$ task $\in$ Tspec task $=(t i d, \operatorname{InD}$, OutD, ExecF,MaxPay $)$, where $t i d$ is the task name; $\operatorname{InD}$ is the data set to be served by workplace; OutD is the output data set after the task has been completed; ExecF is the set of functions to be served by workplace; MaxPay is the maximum allowable payment to service;

- $a \log$ is the work $\log$ recording the migrating, cooperating and security events;

- aEng is the coded engine including task scheduling, service using, migration, security protection, etc.

Definition 2.2 Workplace is defined as $w p=($ pid, Rservs, Tservs, $p E n g)$, where

- pid is the workplace identifier;

- Rservs is the runtime services for mobile agent, such as migration, communication, service recommendation, etc;

- Tservs is the task services $\forall w s \in T$ servs and $w s=(\operatorname{sid}, \operatorname{ServF}, \operatorname{Serv} D, \operatorname{Serv} C)$, sid is the service name; $\operatorname{ServF}$ is the service functions; $\operatorname{Serv} D$ is the service data; ServC is the service fees defined on $\operatorname{ServF}$ and $\operatorname{ServD}$ according to local service rules;

\footnotetext{
1 If the process includes parallel tasks, we can divide the process into a group of branches and each branch is only consisting of sequential tasks.

2 If two adjacent tasks can be finished at the same workplace, we should combine them as one in the process definition stage.
}

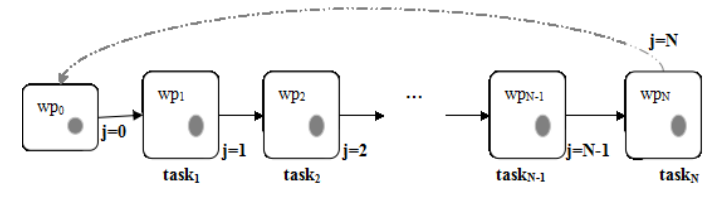

Fig. 1: The path is $w p_{0} \rightarrow w p_{1} \rightarrow w p_{2} \rightarrow \ldots w p_{N-1} \rightarrow w p_{N}$

- $p E n g$ is the service engine, which provides runtime services and task services for mobile agent as required.

Let WP denote the set of all workplaces in the environment.

Definition 2.3 Given task $\in T$ spec and $w p \in W P, w p$ is an available workplace for task if and only if $\exists w s \in T$ servs can make task.ExecF $\subseteq$ ws.ServF $\wedge$ task.InD $\subseteq$ ws.Serv $D \wedge$ task.MaxPay $\leq w s$.ServC being true.

Definition 2.4 For Tspec $=\left(\text { task }_{i}\right)_{i=1}^{N}$, the mobile agent path is defined as path $=\left(w p_{j}\right)_{j=0}^{N}$, where $w p_{0}$ is the launching and returning place of mobile agent. Except for $w p_{0}, w p_{i}$ is the only workplace where $t a s k_{i}$ can be finished, $i=1,2, \ldots, N$.

Fig.1 gives an illustration of definition 2.4, where $j=0,1,2, N$ indicate the order while mobile agent leaves $w p_{0}, w p_{1}, \ldots, w p_{N}$ respectively. In convention, the leaving order is also called the migrating-out time and $w p_{j}$ is named as the current workplace of mobile agent at time $j$.

Let $g_{\text {agent }}^{\text {task }_{i}}$ be the expected gain of task $k_{i} c_{w p_{i}}^{\text {ask }_{i}}$ be the cost executing task $k_{i}$ on $w p_{i}, c_{w p_{i}}^{w p_{i-1}}$ be the cost moving from $w p_{i-1}$ to $w p_{i}$. The one-step gain of mobile agent to move from to can be represented by

$$
r\left(w p_{i} \mid w p_{i-1}\right)=g_{\text {agent }}^{\text {task }_{i}}-c_{w p_{i}}^{\text {task }_{i}}-c_{w p_{i}}^{w p_{i-1}}
$$

The total gain on the path is

$$
G_{p a t h}=\sum_{i=1}^{N} r\left(w p_{i} \mid w p_{i-1}\right)
$$

According to Equation (2), the objective of path planning is to maximize $G_{\text {path }}$ through workplace choice at each migrating-out time.

\section{Path planning model based on MDP}

Let $\Omega_{j}$ be the decision space of mobile agent at migrating-out time $j$ with $w p_{j} \notin \Omega_{j}$, where $w p_{j}$ is the current workplace of mobile agent.

Definition 3.1 The process of mobile agent path planning can be defined as a 4-tuple $(S, A, P, R)$, where

$-S=\left\{s_{0}, s_{1}, \ldots, s_{N}\right\}$ is the set of states, $s_{j} \in S$ indicates mobile agent prepares to leave $w p_{j}$ at time $j$;

- $A=\left\{a_{1}, a_{2}, \ldots, a_{M}\right\}$ is the set of actions, $a_{j} \in A$ indicates mobile agent evaluates $w p \in \Omega_{j}$ and decides whether to choose it as $w p_{j+1}$ at time $j$; 


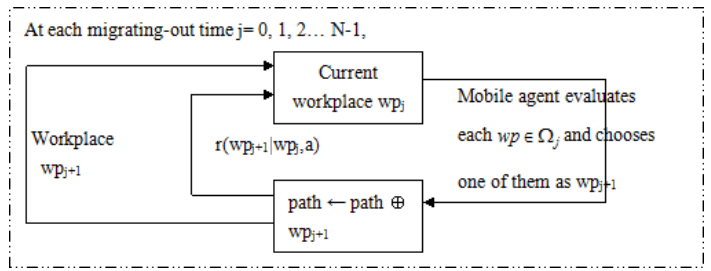

Fig. 2: Path planning model based on MDP. The symbol $\oplus$ represents the linking computation of workplaces.

- $P: S \times A \times S \rightarrow[0,1]$ is the state transfer function, $p\left(s^{\prime} \mid s, a\right)=p\left(w p \mid w p_{j}, a\right)$ indicates the probability of choosing $w p$ as $w p_{j+1}$ by taking the action $a$.

$-R: S \times A \times S \rightarrow R$ is the reward function, indicates the one-step gain of obtained on $w p$ by taking the action $a$.

Fig.2 is an illustration of definition 3.1. At each migrating-out time $j$, mobile agent evaluates all $w p \in \Omega_{j}$ and chooses one of them as $w p_{j+1}$.

According to Definition 3.1 and Equation (2), the objective of path planning is to find the optimal policy $\pi^{*}: S \rightarrow A$ to make sure that mobile agent can get the maximum total gain by choosing workplaces from $\Omega_{j}$ at each migrating-out time $j$, except for $j=N$.

In case of all $\Omega_{j}=W P$ and $W P$ is known, the path planning can be solved with learning method, such as TAP $[13,14,15]$. While in a system with variable $\Omega_{j} \subseteq W P$ and $W P$ is an open environment, it becomes more difficult to use learning method. Therefore we use one-step greedy policy in this paper, that is $\forall j=0,1,2, \ldots, N-1$, the probability of mobile agent to choose $w p \in \Omega_{j}$ as $w p_{j+1}$ at $w p_{j}$ can be expressed as

$$
p\left(w p_{j+1}=w p \mid w p_{j}, a\right)= \begin{cases}1 & \text { if } r\left(w p \mid w p_{j}, a\right)=\underset{w p^{\prime} \in \Omega_{j}}{\operatorname{MAX}}\left\{r\left(w p^{\prime} \mid w p_{j}, a\right)\right\} \\ 0 & \text { otherwise }\end{cases}
$$

The method to build the variable $\Omega_{j}$ based on social acquaintance recommendation was given in the next section.

\section{Method to build variable decision space}

Suppose that every social member in the real world, whatever organization or individual,is rational and can be labeled by element of $\Sigma=1,2 \ldots M$. We use $1,2, \ldots, M$ to identify social members. The social role theory [16] shows that every social member plays a social role and possesses social acquaintance relationships consistent with the role, such as client-servant relationship, etc.

Denoting the acquaintance relationship between members $i$ and $j$ as $\operatorname{SAR}(i, j)$ and it is considered as a symmetric relationship.

Property 4.1 The acquaintance relationship has the following properties:

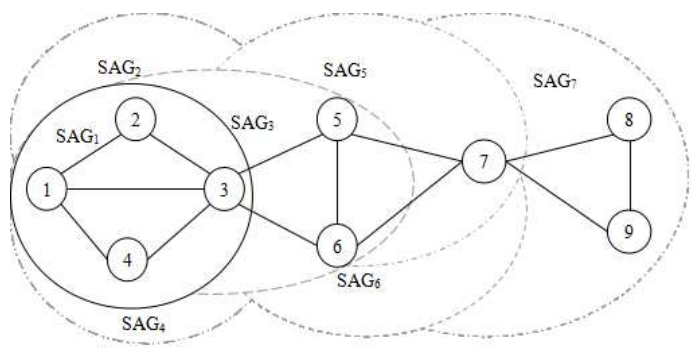

Fig. 3: An example of social acquaintance network and social acquaintance domain.

1. Randomness. Since there is uncertainty in the occurrence of cooperative events, $\operatorname{SAR}(i, j)$ is a random variable.

2. Convergence. If $S A R(i, j) \wedge S A R(i, k)$ then the member

$i$ has both direct acquaintances $j$ and $k$.

3. Transitive. If $\operatorname{SAR}(i, j) \wedge \operatorname{SAR}(j, k)$ then the member $\mathrm{i}$ has indirect acquaintance $\mathrm{k}$.

Definition 4.1 Social acquaintance network is defined as $S O A-N e t=(\Sigma, E)$, where $\Sigma$ is the set of social members, $i \in \Sigma$ represents the social member $i$; $E$ is the set of edges, $e(i, j) \in E$ shows the direct acquaintance relationship $\operatorname{SAR}(i, j)$.

Property 4.2 SOA-Net is a random network, and there are no isolated nodes or sub-networks.

Since $\operatorname{SAR}(i, j)$ is random, $e(i, j)$ is random and $S O A-N e t$ is a random network. The assumption of rational social members implies that there is no isolated node in SOA-Net, and the convergent and transitive properties of acquaintance relationship ensure that there is no insolated sub-network in SOA-Net.

Definition 4.2 For $i \in$ SOA-Net, $S A G_{i}=\left.\{x\}\right|_{e(i, x) \in S O A-N e t}$ is an acquaintance group of $i$, and naming $i$ as the master of $S A G_{i}, x$ as the group member of $S A G_{i}$.

Acquaintance group is the result of the convergence of acquaintance relationship. The degree of node $i$ in $S O A-N e t$, denoted as $\delta_{i}$, equals to the number of group members of $S A G_{i}$. Fig. 3 is an example of acquaintance groups.

Definition 4.3 Let $i \in S O A-N e t$ be the current service member of mobile agent and task $\in$ Tspec to be executed, direct acquaintance recommendation refers to the process that $i$ recommends service members in $S A G_{i}$ for task, while indirect acquaintance recommendation refers to the process that $x \in S A G_{i}$ is entrusted to recommend service members in $S A G_{x}-\{i\}$ for task with $S A G_{x} \not \subset\{i\} \cup S A G_{i}$, and naming $x$ as the trustee.

Take Fig.3 as an example, here $S A G_{1}=\{2,3,4\}$. Since $S A G_{2}=S A G_{4}=\{1,3\} \subset\{1\} \cup S A G_{1}$, there is no new member in SOA-Net that can be recommended by 2 and 4, they do not have to become trustee of 1 . As $S A G_{3}=\{1,2,4,5,6\} \not \subset\{1\} \cup S A G_{1}, 3$ can be entrusted to recommend service members in $S A G_{3}-\{1\}$. Because 


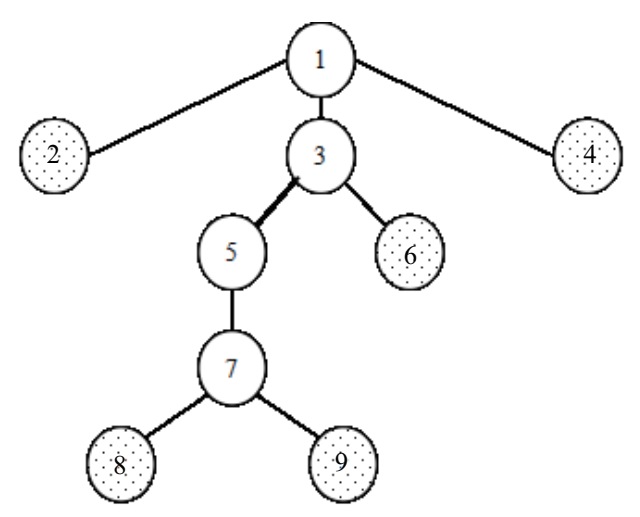

Fig. 4: Acquaintance recommendation tree based on Fig.(3).

two adjacent tasks in Tspec will not be executed in the same workplace, as a result, 3 do not need to check the service of 1 for the task.

Property 4.3 Indirect acquaintance recommendation can be transitively executed in SOA-Net.

Since every trustee may become a new master due to the transitive property of acquaintance relationship, indirect acquaintance recommendation can be transitively executed in SOA-Net.

Taking example of Fig.3, since $S A G_{5}=\{3,6,7\} \quad \not \subset \quad\{3\} \cup S A G_{3} \quad$ and $S A G_{6}=\{3,5,7\} \not \subset\{3\} \cup S A G_{3}, 3$ is able to pass the task to 5 and 6 . However, entrusting duplication will happen if the task is passed from 5 to 6 or from 6 to 5 . Similarly, it will result in the same thing if both 5 and 6 pass task to 7 . To avoid entrusting duplication, we adopt tree policy in this paper. An acquaintance recommendation tree corresponding to Fig.3 is shown in Fig.4, in which the root represents the current service member 1, the leaf for the members who do not need to be entrusted, and the middle nodes for the members who are both trustee and masters.

Let $i \in S O A-N e t$ be the current service member of mobile agent, the acquaintance tree of $i$ is denoted as $A$ Tree $_{i}$, where $i$ is the root node, and $\forall x \in S A G_{i}, x$ is the leaf node.

Definition 4.4 Given $i \in S O A-N e t$, the acquaintance recommendation tree of $i$, denoted as RecTree, is a tree recursively defined in the following way,

1. ATree $_{i}$ is a RecTree;

2. Suppose $x$ is a leaf node on RecTree, ATree $_{x}$ is a subtree on RecTree if and only if

(a) $\forall y \in$ ATree $_{x}$, if $y \in \operatorname{Rec}$ Tree then cut $\mathrm{y}$ from ATree $_{x}$, and

(b) The set of leaf nodes ATree $_{x}$ is not empty.

Definition 4.5 Let $i \in S O A-N e t$ is the current service member of mobile agent and task $\in$ Tspec is the task to be executed, the service recommendation refers to the process that $i$ recommends service members in $S A G_{i}$ for task at first, and then transitively executes indirect acquaintance recommendation along $\operatorname{RecTree.}$

For task $\in T$ spec, let $\Omega_{\text {task }}$ be the set of members created through service recommendation.

Property 4.4 $\forall$ task $\in$ Tspec, $\Omega_{\text {task }}$ varies with task.

According to Definition 4.2, $\forall i \in S O A-N e t, \delta_{i}$ varies and group members in $S A G_{i}$ are distributed unevenly in SOA-Net. As a result, $\Omega_{\text {task }}$ varies with task. The more workplaces there is in $\Omega_{\text {task }}$, the higher probability mobile agent are able to find the optimal service.

Theorem 4.1 Given Tspec, mobile agent is able to dynamically construct path by way of service recommendation.

Proof: $\forall$ task $\in T$ spec, if task can be completed in social world $\Sigma$, there must be $\Omega_{\text {task }}(\neq \Phi)$ in SOA-Net. Since there is no isolated node or sub-network in SOA-Net, if $\Omega_{\text {task }}$ exists, it must be able to be found via service recommendation. Thus, mobile agent can choose service members from $\Omega_{\text {task }}$ definitely. That is, mobile agent is able to dynamically construct its path.

$\forall i \in \Sigma$, Supposing that $i$ can provide one and only one workplace $w p_{i}$ for mobile agent, SOA-Net can be mapped into a homogeneous workplace network.

Definition 4.6 Workplace network is defined as $W P-N e t=(W P, E), W P$ is the set of workplaces, $i \in S O A-N e t \rightarrow w p_{i} \in W P-N e t ; E$ is the set of edges, $e(i, j) \in S O A-N e t \rightarrow e\left(w p_{i}, w p_{j}\right) \in W P-N e t$.

According to Definition 4.6, WP-Net inherits all properties of SOA-Net. In the following discussion, the mapped concepts will be used without redefining.

\section{Path planning algorithm}

Let $w p$ be the current workplace of mobile agent and task is the task to be executed. Path planning is done in two steps: (1) Mobile agent inquires $w p$ to recommend decision space $\Omega_{\text {task }}$; (2) Mobile agent chooses the optimal one from $\Omega_{\text {task }}$ as new workplace.

Since $W P-N e t$ is a huge and open network, considering the execution efficiency, generally mobile agent will not request $w p$ to recommend all the service-satisfied workplaces in WP-Net. In order to control the recommendation process, let $\lambda$ be the minimum number of workplaces for all $\Omega_{\text {task }}$.

Let $A T r e e_{w p}$ be the acquaintance tree of $w p, \delta$ be the number of leaf nodes on ATree ${ }_{w p}$, RecTree be the recommendation tree of $w p$, and initializing RecTree $=$ Atree $_{w p}, \Omega_{\text {task }}=\Phi$.

The basic idea of algorithm 1 is: Firstly, $w p$ recommends all the workplaces that can satisfy task in $S A G_{w p}$. If $\left|\Omega_{\text {task }}\right|<\lambda, w p$ will pass the task to its group members along RecTree, and each trustee will recommend all the workplaces that can satisfy task in their own acquaintance group, ..., until $\Omega_{\text {task }} \geq \lambda$. 


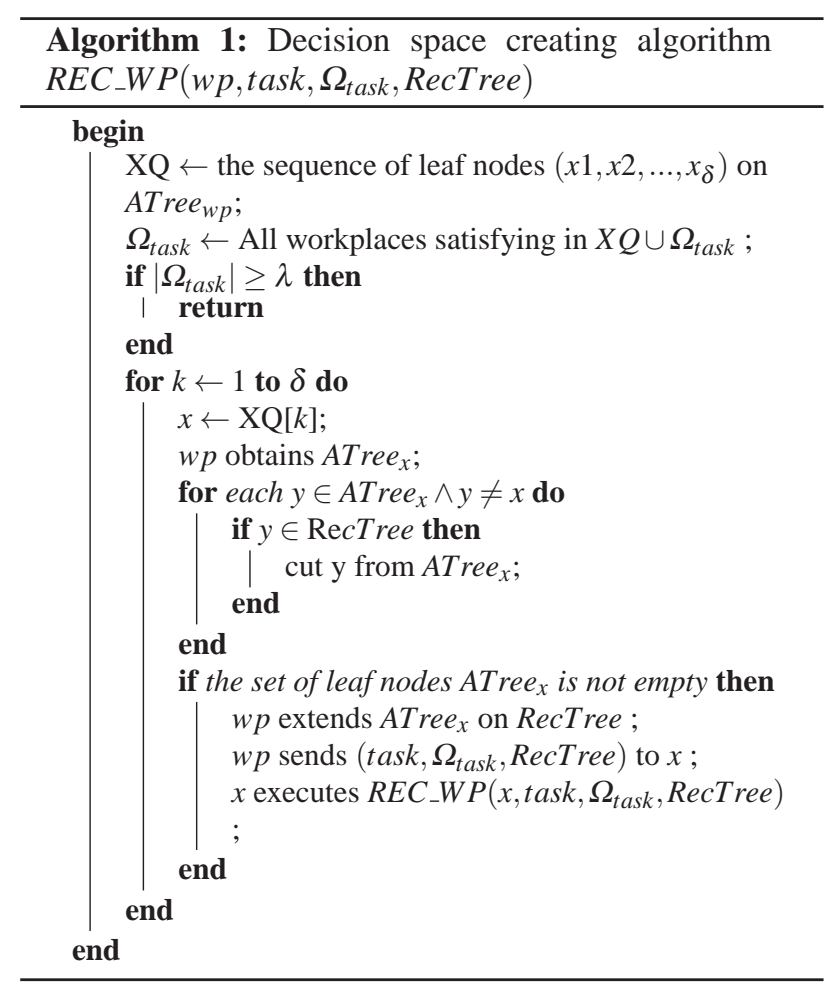

If the communication cost in passing task is neglected, the time cost of algorithm 1 would be

$$
\text { Time }_{\Omega_{\text {task }}}=\sum_{j=1}^{n} \delta_{j}
$$

of which, $n$ is the number of workplaces that took part in recommendation process for creating $\Omega_{\text {task }}, \delta_{j}$ is the number of times that workplace $j$ compared task and services, numerically it equals to the number of group members in $S A G_{j}$.

It needs to be pointed out that, service recommendation may cause duplicate evaluation of the same workplace if it is a shared group member of two master workplaces in WP-Net. Considering that each social member is an autonomous entity, and its acquaintance knowledge is independent, algorithm 1 didnt exclude duplicate evaluation.

Let $g_{\text {agent }}^{\text {task }}$ be the expected gain of the task, MaxG be the maximum gain actually obtained from $\Omega_{\text {task }}$, and NextWP $\in \Omega_{\text {task }}$ be the workplace corresponding to MaxG.

The basic idea of algorithm 2 is that mobile agent obtains $\Omega_{\text {task }}$ firstly, and then chooses the workplace with maximum gain as the new one. If we neglect the time spent in communication to obtain $c_{w p_{j}}^{\text {task }}$ and $c_{w p_{j}}^{w p}$, the factors affecting the time complexity of algorithm 2 are obtaining $\Omega_{\text {task }}$ and choosing the optimal workplace from $\Omega_{\text {task }}$. The time cost of the former can be calculated with Equation (4), and the number of comparison of the latter is $\left|\Omega_{\text {task }}\right|$.

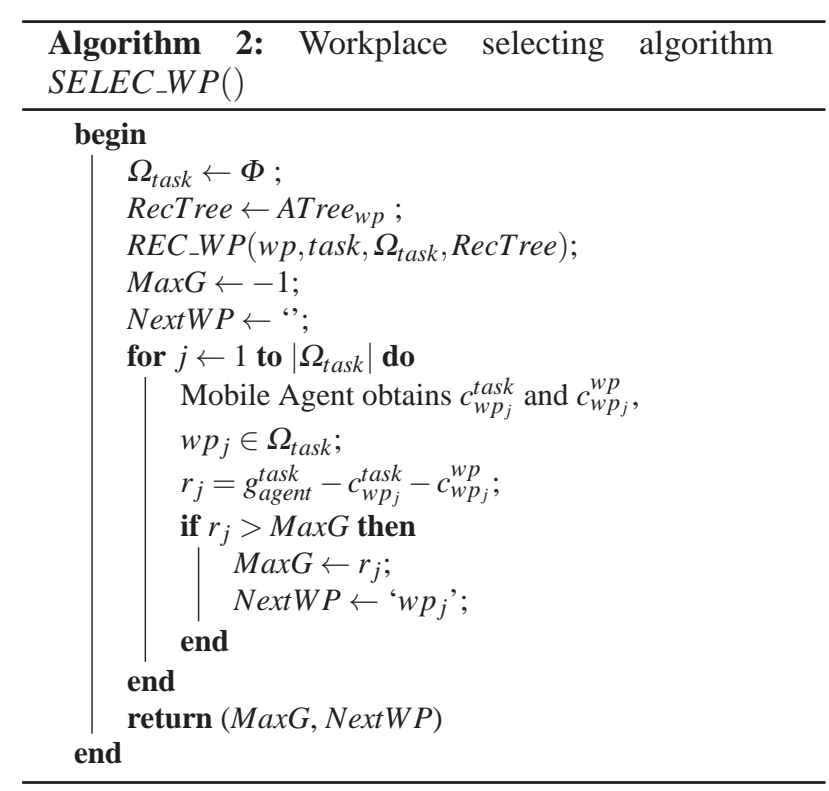

\section{Experiment and analysis}

To simplify the calculation, $\forall$ task $\in$ Tspec, assuming that the satisfaction degree of task can be denoted by the expected gain $r_{\text {agent }}^{\text {task }}=\theta$, where $\theta$ is a non-zero constant.

\subsection{Arrangement of experiment environment}

The experiment environment was set with the following method.

1. Generate $W P-N e t$

(a)Randomly generate a set of $M$ points in the twodimensional space as $W P=\{w p\}_{1}^{M}$.

(b) $\forall w p_{i}, w p_{j} \in W P \wedge i \neq j$,connect $w p_{i}$ and $w p_{j}$ in the probability of $\beta \times \exp \left[\operatorname{rand}(0,1) \frac{-M}{\mu}\right]$, where $\mu \geq 1$ is the expected value of degrees of nodes, and $0<\beta<1$ is the node connection control parameter[17].

2. Generate $\Omega_{\text {task }}$

(a) $\forall$ task $\in$ T spec randomly choose $\lambda$ workplaces but $w p$ in WP-Net as $\Omega_{\text {task }}, w p$ is the current workplace of mobile agent.

(b) $\forall w p_{k} \in \Omega_{\text {task }}$ generate a random number $\operatorname{rand}[\theta, 1.5 \theta]$ for $w p_{k}$ as the gain $r_{w p_{k}}^{\text {task which }}$ mobile agent can obtain at $w p_{k}$ actually, where $\theta$ is the same as in $r_{\text {agent }}^{\text {task }}$.

\subsection{Total gain and efficiency of path planning}

According to Algorithm 2, mobile agent always chooses the workplace with maximum gain from $\Omega_{\text {task }}$ as the 
workplace where task is executed, the total gain on the path can be calculated with Equation (5),

$$
G=\sum_{i=1}^{N}\left(r_{w p}^{\text {task }_{i}}=\underset{w p^{\prime} \in \Omega_{\text {task }_{i}}}{\operatorname{MAX}}\left\{r_{w p^{\prime}}^{\text {task }_{i}}\right\}\right)
$$

Omitted the cost of entrusting recommendation, the Equation (6) is used to represent the efficiency of path planning, of which, Time $\Omega_{\text {task }_{i}}$ be calculated with Equation (4). The larger $\eta$ is, the less efficient of planning is.

$$
\eta=\frac{1}{N} \sum_{i=1}^{N} \text { Time }_{\Omega_{\text {task }}}
$$

\subsection{Experiment results and analysis}

The objective of experiment is to examine the effect of the changes of environment parameter $M$ and $\lambda$ on the total gain $G$ and the path planning efficiency $\eta$.

Suppose $N=20, \theta=5, \mu=5$ and $\beta=0.5$.

The process of generating path for $N$ tasks is named as one time of experiment. For the given $M$ and $\lambda$, all the results are the average values of 30 experiments and all of which are done based on the same WP-Net, but $\Omega_{\text {task }}$ and $r_{w p_{k}}^{\text {task }}\left(w p_{k} \in \Omega_{\text {task }}\right)$ are generated randomly.

Fig 5. shows $G$ and $\eta$ as $\lambda$ changes while $M$ is constant. The larger $\lambda$ is, the greater $G$ is, the larger $\eta$ is.

When $\lambda$ risen, $\left|\Omega_{\text {task }}\right|$ increased, and the number of workplaces with greater gain increased as well. Since mobile agent always selects the workplace with the greatest gain from $\Omega_{\text {task }}$, the $G$ increased, as shown in the left part of Fig.5 . In the right part of Fig.5, when $\lambda$ became larger, $\left|\Omega_{\text {task }}\right|$ increased, $\eta$ also increased.

Fig. 6 shows $G$ and $\eta$ as $M$ changes while $\lambda$ is constant. The larger $M$ is, the larger $\eta$ is, while $G$ basically stays constant.

Since $\Omega_{\text {task }}$ is randomly generated in WP-Net, when $\lambda$ is constant while $M$ increases, the probability of $\Omega_{\text {task }}$ to be sparsely distributed in WP-Net will increase, and the number of workplaces that took part in recommendation process for creating $\Omega_{\text {task }}$ and the number of times comparing task and service increases as shown in the right part of Fig.6. Since Equation (5) is independent from $M$, if $\lambda$ stays constant, the workplace gain in $\Omega_{\text {task }}$ can only be affected by random number $\theta \leq r_{w p}^{\text {task }} \leq 1.5 \theta$. As shown in the left part of Fig. 6 , the average result of 30 experiments makes the curve stable despite some fluctuation.

Fig. 7 shows $G$ and $\eta$ as $M$ and $\lambda$ changes. The larger $M$ and $\lambda$ is, the larger $\eta$ is. However the total gain on the path becomes stable and close to the maximum one.

As shown in Fig.5 to Fig.7, the key parameter that affects $G$ and $\eta$ is $\lambda$. If the goal of path planning is to pursue maximum gain, $\lambda$ should be larger. If the goal of planning is to pursue maximum efficiency, $\lambda$ should be smaller. However if $\left|\Omega_{\text {task }}\right|$ is too small, the adaptability will reduce due to the lack of services.

\section{Related works}

The problem of mobile agent path planning can be solved in static or dynamic manner.

Static planning is the approach that path is created before mobile agent has been launched. The most commonly used static planning methods are itinerary design and path learning with known WP. Itinerary design method concludes the migrating behavior of mobile agent as structured reusable itinerary patterns, such as sequence, branch and parallel. Itinerary pattern can be described with itinerary languages [3] or itinerary algebra [4]. Since itinerary patterns cannot cover the ad hoc migrating behavior of mobile agent, the static itinerary cannot meet the environment changes. Although itinerary with redundant workplaces $[18,19,20]$ can provide some flexibility to the workplace selection, but the redundant workplace information is a prior knowledge, the adaptability is still limited. The path learning approach regards the environment was fixed and known, such as TAP [13,14,15], genetic [21] and swarm [22,23, 24,25] algorithms. As compared with itinerary design, the advantage of path learning is to be able to use the whole network information automatically. However the network information used in the learning algorithm is the historical information before mobile agent was launching, as a result, the path generated cannot adapt well to the environment changes during the mobile agent implementation. TAP $[13,14,15]$ also describes the path planning issue as MDP with a fixed and known environment. Since the basic idea of TAP is derived from TSP [26], each workplace appears only once in the path. The MDP model introduced in this paper allows decision space vary for deferent tasks, therefore it brings more flexibility to path planning.

Dynamic planning refers to the approach that mobile agent has no path information before it starts to migrate. Every time it migrates, the new workplace is selected based on its goal and knowledge of the current environment. Compared to static planning, the advantage of dynamic planning is high adaptability to an open environment, while the disadvantage is that it takes a long time to understand the environment, therefore decreasing efficiency. The way of obtaining knowledge of the current environment can be divided into service discovery and service recommendation. Examples for the former are PHA*[6], LCF and GCF [7,8], etc. Since service discovery requires mobile agent to carry sufficient knowledge and coded functions with it, it is possible that mobile agent be large, and migration delay and fault will happen. The example for the latter is navigating mechanism [11]. Compared to the service discovery, navigating can make mobile agent lightweight, but constructing the unified navigating tree requires prior knowledge of whole environment and it cannot support an open environment either.

The acquaintance recommendation method introduced in this paper is similar to the navigation from 

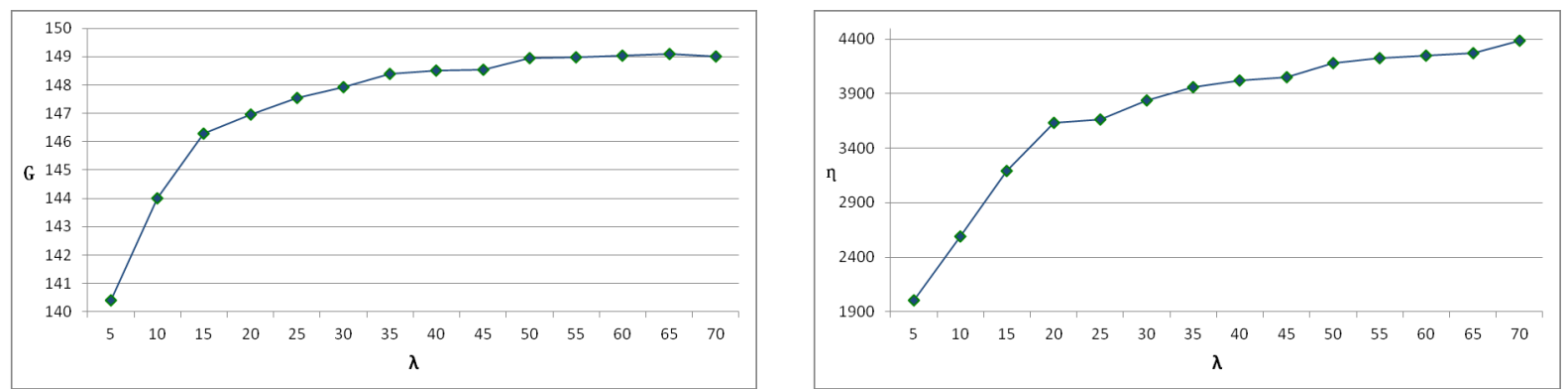

Fig. 5: Changes of $G$ and $\eta$ as $\lambda$ increase, $M=1000$.
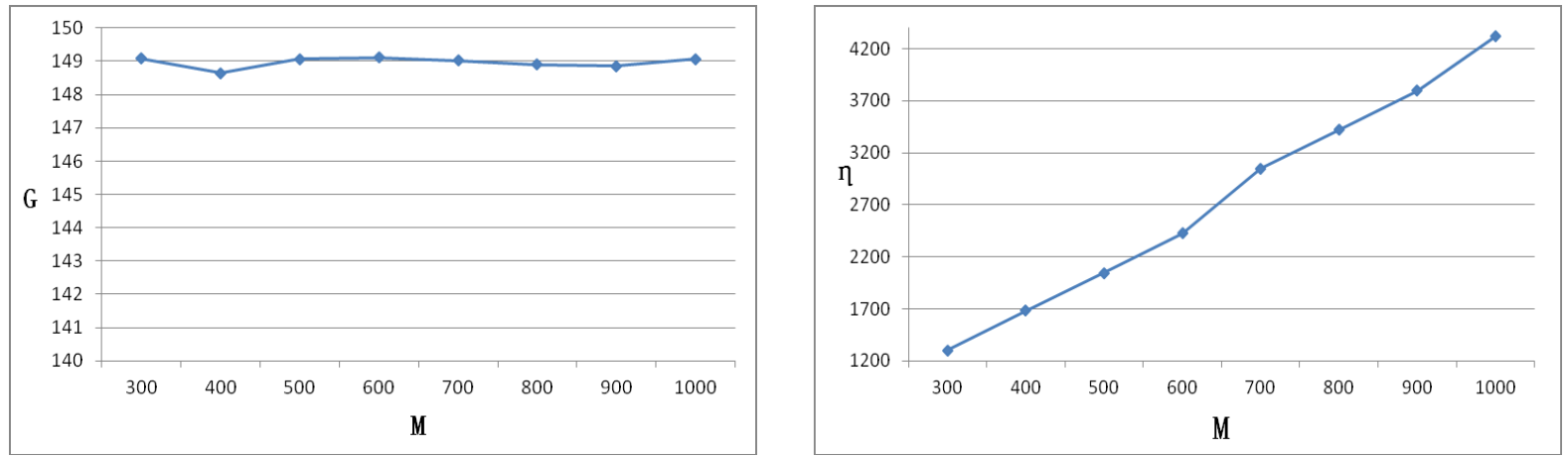

Fig. 6: Changes of $G$ and $\eta$ as $M$ increase, $\lambda=60$.
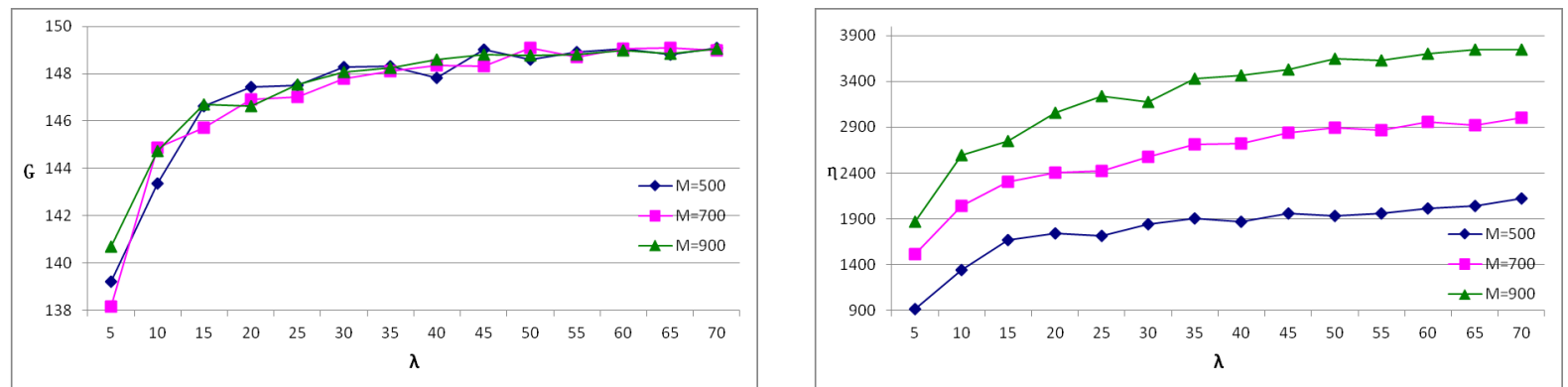

Fig. 7: $G$ and $\eta$ as $M$ and $\lambda$ change

the service recommendation perspective. Both of them can make mobile agent lightweight. The acquaintance recommendation mechanism is implemented based on an open social network and does not rely on the whole environment. As long as cooperation between social members is rational, mobile agent can always use acquaintance recommendation to dynamically planning its path.

\section{Conclusions}

The main contribution of this paper is proposed a dynamic planning method for mobile agent implementation based on the variable decision space. The path planning model is described with MDP, and the variable decision space is generated through social acquaintance recommendation. Since both mobile agent and workplace are proxies of the social members, as long as the goal of mobile agent can be achieved in the social network, mobile agent is constantly be able to dynamically construct its path with the method introduced in this paper. The experiment results and analysis show that the larger the decision space recommended to mobile agent is, the greater the total gain can be obtained, and the less efficient the planning is.

Social acquaintance recommendation provides a feasible way for mobile agent path dynamic planning. 
The future work mainly includes: acquaintance recommendation with constrains of time and payment, multi-agent path planning based on social acquaintance recommendation, etc.

\section{Acknowledgement}

The work presented this paper is supported by the National Science Foundation of China (Grant No.60573169,61173068) and the Natural Science Foundation of Shandong Province of China (Grant No. ZR2009GM021).

\section{References}

[1] R. Bellman, Dynamic Programming, Princeton University Press, Princeton, New Jersey, (1957).

[2] R. S. Sutton and A. G. Barto, Reinforcement Learning: An Introduction, MIT Press, Cambridge, MA, (1998).

[3] Y. Aridor and D. B. Lange, Agent Design Patterns: Elements of Agent Application Design, Proceedings of the second international conference on Autonomous agents, Minneapolis, Minnesota, USA, 108-115 (1998).

[4] D. Chacn, J. McCormick, S. McGrath, and C. Stoneking, Rapid Application Development Using Agent Itinerary Patterns, Technical Report \#01-01, Lockheed Martin Advanced Technology Laboratories, March, (2000).

[5] S. W. Loke, H. Schmidt, and A. Zaslavsky, Programming the Mobility Behavior of Agents by Composing Itineraries, Lecture Notes in Computer Science, 1742, 214-226 (1999).

[6] A. Felner, R. Stern, S. Kraus, and A. Ben-Yair, PHA*: Finding the Shortest Path with A* in An Unknown Physical Environments, Journal of Artificial Intelligence Research, 21,631-679 (2004).

[7] H. Qi and F. Wang, Optimal Itinerary Analysis for Mobile Agents in Ad Hoc Wireless Sensor Networks, Proceedings of the IEEE 2001 International Conference on Communications, Helsinki, Finland, 147-153 (2001).

[8] M. Chen, V. Leung, S. Mao, T. Kwon, and M. Li, Energy-efficient Itinerary Planning for Mobile Agents in Wireless Sensor Networks, Proceedings of the IEEE 2009 International Conference on Communications, Bresden, Germany, 1-5 (2009).

[9] P. E. Hart, N. J. Nilsson, and B. Raphael, A Formal Basis for the Heuristic Determination of Minimum Cost Paths, IEEE Transactions of Systems Science and Cybernetics, 4, 100-107 (1968).

[10] L. Rech, C. Montez, and R. S. Oliveira, Itinerary determination of imprecise mobile agents with firm deadline, An International Journal of Web Intelligence and Agent Systems, 6, 421-439 (2008).

[11] J. Cheng, G. Z. Zeng, and H. He, Research on Migrating Instance Path Planning in Migrating Workflow System, Journal of Frontiers of Computer Science and Technology, 2, 658-665 (2008).

[12] J. L. Gao and G. Z. Zeng, Research of Mobile Agent Workflow Path Dynamic Programming Method Based on Social Acquaintance Network,3rd International Conference on Information Sciences and Interaction Sciences, 398-402 (2010).

[13] K. Moizumi, Mobile Agent Planning Problem, Ph.D. dissertation, Dartmouth College, Hanover, NH, (1998).

[14] K. Moizumi and G. Cybenko, The Traveling Agent Problem, Mathematics of Control, Signals and Systems, 14, 213-232 (2001).

[15] J. W. Baek, G. T. Kim, and H. Y. Yeom, Timed Mobile Agent Planning for Distributed Information Retrieval, Proceedings of the International Conference on Autonomous Agents, Montreal, Quebec, Canada, 120-121 (2001).

[16] B. J. Biddle, Role Theory: Expectations, Identities, and Behaviors, New York, Academic Press, (1979).

[17] K. J. Liu, Z. W. Yu, and Z. Q. Cheng, Study on Model for Stochastic Network Simulation and Its Key Techniques, Computer Engineering and Applications, 41, 130-132/217 (2005).

[18] K. Koukoumpetsos and N. Antonopoulos, Agent NavigatorA Model for Dynamic, Flexible Agent Mobility, Proceedings of the International Conference on Parallel and Distributed Processing Techniques and Applications, Las Vegas, Nevada, USA, (2000).

[19] J. W. Baek, J. H. Yeo, and H. Y. Yeom, Agent Chaining: An Approach to Dynamic Mobile Agent Planning, 22nd IEEE International Conference on Distributed Computing Systems, Vienna, Austria, 579-586 (2002).

[20] D. Y. Liu, B. Yang, K. Yang, and S.S. Wang, Migration Strategies of Mobile Agent Based on Itinerary Graph, Journal of Computer Research and Development, 40, 838-845 (2003).

[21] S. H. Piao and B. R. Hong, A Path Planning Approach to Mobile Robot under Dynamic Environment, ROBOT, 25, 1821/43 (2003).

[22] J. Jiao, S. Yao, and C. Xia, Application for Artificial Bee Colony Algorithm in Migration of Mobile Agent, Communications in Computer and Information Science, 93, 232-238 (2010).

[23] J. Cheng and G. Z. Zeng, A Greedy Particle Swarm Optimization Algorithm for Workplace Planning in Migrating Workflow, Fourth International Conference on Natural Computation, 6, 407-411 (2008).

[24] Y. W. Feng, Z. Q. Wei, and X. P. Ji, A MA Sub-optimal Migration Strategy based on Probability, Journal of Harbin Institute of Technology, 40, 1815-1819 (2008).

[25] J. Li, Y. Chai, P. Li, and H. Yin, Multi-cellular-ant Algorithm for Large Scale Capacity Vehicle Route Problem, Advances in Swarm Intelligence, Lecture Notes in Computer Science, 6728, 260-266 (2011).

[26] G. Laporte, The Traveling Salesman Problem: An Overview of Exact and Approximate Algorithms, European Journal of Operational Research, 59, 231-247 (1992). 


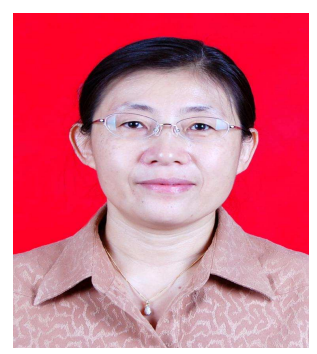

Xiaolin Wang is
currently an associate
professor in School of
Computer Science and
Technology of Shandong
University. Her research
interests include mobile
computing and its application
technology, CSCW and
workflow management.

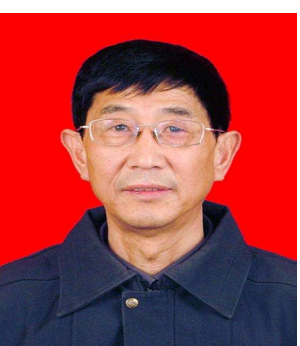

Guangzhou

Zeng

is currently a professor, Ph.D. supervisor. His research interests include CSCW, intelligent computing theory and technology, mobile computing and its application technology. 